\title{
A new Mean Deviation and Advanced Mean Deviation Techniques to Solve Multi-Objective Fractional Programming Problem via Point-Slopes Formula
}

\author{
Rebaz B. Mustafa ${ }^{1}$, Nejmaddin A. Sulaiman ${ }^{2}$
}

* Corresponding Author

1 Department of Management Information System, Erbil Technical Administrative Institute, Erbil Polytechnic University, Erbil, Iraq, rebaz.mustafa@epu.edu.iq 2 Department of Mathematics, College of Education, Salahaddin University, Erbil, Iraq, nejmadin.sulaiman@su.edu.krd

\begin{abstract}
In this paper, we have proposed an alternate new technique to find an efficient solution of multi-objective fractional programming problems (MOFPP). The multi-objective fractional programming problem will be converted into multi-objective linear programming (MOLPP) utilizing the point-slopes formula, which has equivalent weights to the original MOFPP. The MOLPP will be diminished to a single objective linear programming problem (SOLPP) through using two new techniques for the values of the objective function and suggesting an algorithm and flow chart for its solution. Finally, we obtained the optimal solution for MOFPP by solving the consequent linear programming problem (LPP) based upon simplex method. The proposed practicability is confirmed with the existing approaches, and it has been illustrated with some numerical examples. The new techniques outperform other techniques in terms of results, with a smooth pace, and reach an efficient solution at a faster rate.
\end{abstract}

Key Words: Multi-Objective Linear Fractional Programming Problem, Multi-Objective Quadratic Fractional Programming Problem, Linear Programming Problem, Point-Slopes Formula, Mean Deviation.

Mathematical Subject Classification: 90C05, 90C29, 90C30, 90C32

\section{Introduction}

In the past five decades, the fractional programming problem (FPP), which has been utilized as a significant designing tool, has been exercised in various disciplines, for instance, business, manufacturing planning, economic and corporate organization, health care, and hospital planning, etc. FPP arises repeatedly in decision making implementations, including game theory, transportation, etc. Fractional programming is commonly applied for modeling real-world issues with one, two or more objective(s) for example, profit over cost, stocking/sales, cost/ time, etc. The idiom multi-objective programming is used to imply a kind of optimization problem where two or more objectives are to be maximized, minimized, or both of them subject to definite constraints. Usually, to resolve or suggest new methodology issues, we will be able to provide some previous facts and concepts. It has been described in section three. Also, for different kinds of FPP, there are some distinct sorts of research. Some of them compact with theory, (Borza \& Rambely, 2021; Hejazi \& Nobakhtian, 2020) or some of them methods of solution with applications(Akhtar et al., 2017; Pramy \& Islam, 2017; Suleiman \& Nawkhass, 2013) and many researchers have studied how to convert MOFPP into LPP, using several methods and techniques, such as Chakraborty and Gupta's approach(Chakraborty \& Gupta, 2002), Dinkelbach's methodology(Dinkelbach, 1967), and Nayak, \& Ojha (Nayak \& Ojha, 2019) etc. The aim of the paper is to present a new efficient technique for remediation of the MOFPP, with fewer steps and suitable precision to convert the MOFPP into LPP. In this paper, a new model has been suggested for solving MOFPP always yields an efficient solution and reduces the complexity of solving the MOFPP. The idea of an efficient solution is considered as an alternative of exact optimal solution. A solution is 
efficient if moving to an extra solution does not develop all the objectives. Suitable changes have been applied to express an equivalent MOLPP and the resulting MOLPP has been solved based on the set of techniques. The equivalency has also been established in the feasibility region is affected, and numerical examples have been presented to support the model. This work concentrates on MOFPP, where several fractional objective functions (i.e. the ratio of two linear functions, ratio of two quadratic functions, or the numerator quadratic function and the denominator linear function) are to be optimized together subject to a set of linear inequality constraints and nonnegative decision variables. We have investigated a solution to the MOFPP based on a feasible region, and introduced an algorithm and flowchart to solve it. The MOFPP is changed into a MOLPP by the point-slopes formula. After that, we suggested a new mean deviation and advanced mean deviation techniques be used to tackle the MOLPP reduced into LPP; this LPP is solved by the classical simplex method. The results were compared with different techniques, such as Chandra Sen(Sen, 1983), advanced optimal average(Akhtar et al., 2017), advanced harmonic mean(Akhtar \& Modi, 2017), Chakraborty and Gupta’s approach, and Lachhwani’s approach.

This paper is organized into eight sections following the introduction in section 2 we show the literature reviews. In section 3, we give some definitions correlated to our work, in section 4, we present two forms of the MOFPP, in section 5 we describe in detail our technique to solution with algorithm and flow chart, we present four numerical examples in section 6 , followed by discussion of the results in section 7 , we conclude in section 8 .

\section{Literature Review and Problem Statement:}

In this section, we show and cite some related works and approaches with regards to our trend. In the paper (Dinkelbach, 1967) Dinkelbach offered to explain an algorithm for solving nonlinear programming based on a theorem by Jagannathan. Also (Sen, 1983)Chandra Sen introduced the MOLPP and proposed a technique to solve the MOLPP under the definition that the values are optimal for problems individually when the result is positive. (Chakraborty \& Gupta, 2002)Chakraborty and Gupta presented an equivalent MOLPP form of the multi-objective linear programming problem (MOLFPP) using a fuzzy set theoretic approach. Valipour, Yaghoobi, and Mashinchi (Valipour et al., 2014) suggested an iterative parametric approach to find an efficient solution to the MOLFPP, that extends Dinkelbach's approach, when solving one LPP in each iteration. Noura, Sherafatmand, and Hajihosseini (2013) suggested a new method for solving MOLFPP; the new method is based upon transforming MOLFPP into MOLPP using the Primal-Dual approach. Guzel(Güzel, 2013) suggested a new solution to the MOLFPP based on a theorem previously studied by Dinkelbach and reduced MOLFPP to LPP. Mehdi, Chergui, and Abbas(Mehdi et al., 2014) described an improvement to Cherqui and Maulai's method based on the branch and cut concept, this method consists of fractional objectives over the original feasible set and proposes to linearize one of the fractional objectives to solve LP as subproblems rather than fractional linear programming, which facilitates the use of the Dual Simplex Method. Sulaiman and Mustafa(Sulaiman \& Mustafa, 2016) presented a new technique for transforming MOLPP into LPP using harmonic mean for values of objective functions, with some limitations. Lachhwani(Lachhwani, 2017) studied an alternate modified method for solving multi-objective quadratic fractional programming problems (MOQFPP) based on a fuzzy goal programming approach. Akhter, Modi, and Duraphe(Akhtar et al., 2017) defined an advanced optimal average of maximin and minimax technique to convert MOQFPP into a single objective quadratic fractional programming problem (SOQFPP), but there were unresolved issues related to the value of objectives. Pramy and Islam(Pramy \& Islam, 2017) presented a modified method and tried to find an efficient solution to MOLFPP. Sirvi, Albayrak, and Temelcan(Sivri et al., 2018) studied a novel method that converts quadratic fractional programming problems (QFPP) into LPP using Taylor Series at a chosen initial point. Nayak and Ojha(Nayak \& Ojha, 2019) proposed a method to solve the MOLFPP using a parametric approach. Gupta, Rani, and Goyal(Gupta et al., 2019) proposed an algorithm with an efficient method to obtain a solution of the multi-objective quadratic fractional optimization model (MOQFOM) with a trapezoidal fuzzy number, used $\alpha$ - cut method, although, this idea tried to reduce the error values of the decision. Chandra Sen(Sen, 2020) studied existing averaging techniques and suggested improved averaging techniques applied for solving multi objective optimization (MOO). Hejazi(Hejazi \& Nobakhtian, 2020) proposed the idea of convexificators is used to derive the Karush-Kuhn-Tucker conditions at weak efficient solution of MOLFPP, and investigated the relationships between equality and inequality constraints. Borza and Rambely(Borza \& Rambely, 2021) presented an active methodology for processing the MOLFPP that used the max-min technique to transform MOLFPP into LPP, this technique is one of the best methods for solving higher numbers of objectives in nonlinear programming. In order to solve MOFPP, we have tried to obtain an efficient solution and remove any complexity in the fractional programming. 


\section{Some Related Definitions}

\subsection{Canonical Form of Linear Programming (LP)}

A LP problem may be defined as the problem of maximizing or minimizing a linear function subject to linear constraints. The constraints may be equalities or inequalities. A LP is defined of the form,

Max or $\operatorname{Min}=C^{t} x+\alpha$

Subject to $\operatorname{Ax}(\leq,=, \geq) b, \quad x \geq 0$

Where, $x \in R^{n}$ represents the vector of variables to be determined, $c \in R^{n}$ and $b \in R^{m}$ are vectors of coefficients, $\mathrm{A} \in \mathrm{R}^{\mathrm{n} \times \mathrm{m}}$ is a matrix of coefficients and $\alpha \in \mathrm{R}$ is constant.

\subsection{Vertex:}

A vertex is a corner point of a polygon, polyhedron, or other n-dimensional polytope, in a polygon, a vertex is named convex.

\subsection{Feasible Solution:}

Any set $X=\left\{x_{1}, x_{2}, \ldots, x_{(n+m)}\right\}$ of variables is named a feasible solution of the LP problem, when it accepts the limitations (constraints) (2) and non-negativity boundaries.

3.4 Definition:(Sivri et al., 2018): Let $M$ is a differentiable function, and is continuously differentiable if and only if $\mathrm{M}$ is of differentiability class $\mathrm{C}$, where $\mathrm{M}$ is objective function and $\mathrm{C}$ is a feasible region.

\subsection{Point-Slopes Formula:}

An equation for a plane that contains the point $\left(x_{10}, x_{20}, \ldots, x_{n 0}\right)$ and has slopes $m_{x 1}, m_{x 2}, \ldots, m_{x(n-1)}$ in the $x_{1}, x_{2}, \ldots, x_{n-1}$ directions respectively is

$\left(z=x_{n}\right)-x_{n 0}=m_{x 1}\left(x_{1}-x_{10}\right)+m_{x 2}\left(x_{2}-x_{20}\right)+\cdots+m_{x(n-1)}\left(x_{n-1}-x_{(n-1) 0}\right)$

\subsection{Mean Deviation (MD):}

Mean deviation is the arithmetic mean of the deviations of a series computed from any measure of central tendency, i.e. the mean of the distances of each value from their mean(M). Usually mean deviation is non-negative.

Mean Deviation $(\mathrm{MD})=\frac{\sum_{I=1}^{n}\left(x_{i}-M\right)}{n}$.

Where, $x_{i}=$ the values $(\mathrm{i}=1, \ldots, \mathrm{n}), \mathrm{n}=$ the number of values, and $M=\frac{\sum_{I=1}^{n}\left(x_{i}\right)}{n}$

\section{Multi-Objective Fractional Programming Problem:}

A fractional programming problem with more than one objective is called a multi-objective function, which consists of the ratio of two linear functions, quadratic functions, or numerator quadratic form and denominator linear form, which can be defined as follows:

\subsection{Multi-Objective Linear Programming Problem (MOLFPP):}

That means the numerator and denominator are both linear forms.

$\operatorname{Max} . z_{1}=\frac{c_{1}^{T} \mathrm{x}+\alpha_{1}}{d_{1}^{T} \mathrm{x}+\beta_{1}}$ 
$\operatorname{Max} . z_{2}=\frac{c_{2}^{T} \mathrm{x}+\alpha_{2}}{d_{2}^{T} \mathrm{x}+\beta_{2}}$

$\operatorname{Max} . z_{r}=\frac{c_{r}^{T} \mathrm{x}+\alpha_{r}}{d_{r}^{T} \mathrm{x}+\beta_{r}}$

Min. $z_{r+1}=\frac{c_{r+1}^{T} \mathrm{x}+\alpha_{r+1}}{d_{r+1}^{T} \mathrm{x}+\beta_{r+1}}$

$\cdots$

Min. $z_{S}=\frac{c_{s}^{T} \mathrm{x}+\alpha_{s}}{d_{S}^{T} \mathrm{x}+\beta_{s}}$

Subject to:

$A x\left[\begin{array}{l}\geq \\ \leq \\ =\end{array}\right] b, x \geq 0$

Where $b$ a constant vector is having an m-dimensional, $X$ is a decision variables vector that an $\mathrm{n}$-dimensional, number of maximized objective functions equal to $r$, minimized objective functions equal to (s-r), $r$ and $s$ are positive integer numbers. $A$ is an $(m b y n)$ constants matrix, all vectors are supposed to be pillar except if transposed (T). $c_{i}, d_{i}$ (where $\left.i=1,2, \ldots, \mathrm{s}\right)$ are constants vector have $\mathrm{n}$-dimensional, $\alpha_{i}, \beta_{i}($ where $i=1,2, \ldots, \mathrm{s})$ are scalars.

\subsection{Multi-Objective Quadratic Fractional Programming Problem (MOQFPP):}

We can write two forms of MOQFPP, One of them is with quadratic form over linear form and the other one is with a quadratic form to the numerator and denominator.

Max. $\mathrm{z}_{1}=\frac{\left(c_{11}^{T} \mathrm{x}+\alpha_{1}\right)\left(c_{21}^{T} \mathrm{x}+\beta_{1}\right)}{c_{1}^{T} \mathrm{x}+\gamma_{1}}$ or Max. $\mathrm{z}_{1}=\frac{\left(c_{1}^{T} \mathrm{x}+\alpha_{1}+\frac{1}{2} \mathrm{x}^{T} \mathrm{G}_{1} \mathrm{x}\right)}{\left(d_{1}^{T} \mathrm{x}+\beta_{1}+\frac{1}{2} \mathrm{x}^{T} \mathrm{G}_{2} \mathrm{x}\right)}$
Max. $\mathrm{z}_{2}=\frac{\left(c_{12}^{T} \mathrm{x}+\alpha_{2}\right)\left(c_{22}^{T} \mathrm{x}+\beta_{2}\right)}{c_{2}^{T} \mathrm{x}+\gamma_{2}}$ or Max. $\mathrm{z}_{2}=\frac{\left(c_{2}^{T} \mathrm{x}+\alpha_{2}+\frac{1}{2} \mathrm{x}^{T} \mathrm{G}_{3} \mathrm{x}\right)}{\left(d_{2}^{T} \mathrm{x}+\beta_{2}+\frac{1}{2} \mathrm{x}^{\mathrm{T}} \mathrm{G}_{4} \mathrm{x}\right)}$

Max. $\mathrm{z}_{r}=\frac{\left(c_{1 r}^{T} \mathrm{x}+\alpha_{r}\right)\left(c_{2 r}^{T} \mathrm{x}+\beta_{r}\right)}{c_{r}^{T} \mathrm{x}+\gamma_{r}}$ or Max. $\mathrm{z}_{r}=\frac{\left(c_{r}^{T} \mathrm{x}+\alpha_{r}+\frac{1}{2} \mathrm{x}^{\mathrm{T}} \mathrm{G}_{2 \mathrm{r}-1} \mathrm{x}\right)}{\left(d_{r}^{T} \mathrm{x}+\beta_{r}+\frac{1}{2} \mathrm{x}^{\mathrm{T}} \mathrm{G}_{2 \mathrm{r}} \mathrm{x}\right)}$

Min. $\left.\mathrm{z}_{r+1}=\frac{\left(c_{1 r+1}^{T} \mathrm{x}+\alpha_{r+1}\right)\left(c_{2 r+1}^{T} \mathrm{x}+\beta \mathrm{r}+1\right.}{)}\right)$ or Min. $\mathrm{z}_{r+1}=\frac{\left(c_{r+1}^{T} \mathrm{x}+\alpha_{r+1}+\frac{1}{2} \mathrm{x}^{\mathrm{T}} \mathrm{G}_{2 \mathrm{r}+1-1} \mathrm{x}\right)}{c_{r+1}^{T} \mathrm{x}+\gamma_{r+1}}$

Min. $\mathrm{z}_{s}=\frac{\left(c_{1 s}^{T} \mathrm{x}+\alpha_{s}\right)\left(c_{2 s}^{T} \mathrm{x}+\beta_{s}\right)}{c_{s}^{T} \mathrm{x}+\gamma_{s}}$ or Min. $\mathrm{z}_{s}=\frac{\left(c_{s}^{T} \mathrm{x}+\alpha_{s}+\frac{1}{2} \mathrm{x}^{\mathrm{T}} \mathrm{G}_{2 s-1} \mathrm{x}\right)}{\left(d_{s}^{T} \mathrm{x}+\beta_{s}+\frac{1}{2} \mathrm{x}^{\mathrm{T}} \mathrm{G}_{2 \mathrm{~s}} \mathrm{x}\right)}$

Subject to:

$A x\left[\begin{array}{l}\geq \\ \leq \\ =\end{array}\right] b, x \geq 0$ 
Where, $b$ a constant vector is having an m-dimensional, $X$ is a decision variables vector that an $\mathrm{n}$-dimensional, number of maximized objective functions equal to $r$, minimized objective functions equal to (s-r), $r$ and $s$ are positive integer numbers, and $G_{i}$, is ( $\mathrm{n}$ by $\mathrm{n}$ ) symmetric constant matrix (where $\left.i=1, \ldots, 2 s\right) A$ is an ( $m b y n$ ) constants matrix, every vector is supposed to be column except if transposed $(\mathrm{T}) . c_{1 i}, d_{1 i} c_{i}$, and $d_{i}$ (where $i=$ $1,2, \ldots, \mathrm{s})$ are constants vector have n-dimensional, $\alpha_{i}, \beta_{i}$ and $\gamma_{i}($ where $i=1,2, \ldots, \mathrm{s})$ are scalars.

\section{MOFPP Solution Techniques:}

In this section, the techniques for transforming MOFPP into MOLPP and MOLPP into SOLPP will be explained and studied. The objective functions of MOFPP will be converted into MOLPP utilizing the point-slopes formula. This approach is the only technique that can be used to convert both fractional programming, linear fractional, and quadratic fractional into linear programming of the n-dimension decision variables. In the MOLPP form, it has been generated as an approximation of the MOFPP. Then, the MOLPP can be reduced into a SOLPP by using our techniques concerning useful mean deviation. The merits of this measure are that it is considered to be the better measure of comparison, not much affected by the fluctuations of values, based on an arithmetic mean (average), and it is flexible, because it can be calculated from any average. Although this method has some limitations, for example, when substituting corner points on the feasible region, the denominators of the objective function must not be equal to zero. This method cannot be applied to quadratic programming and linear programming. Meanwhile, the measure has some demerits, such as being not capable of further algebraic dealing. It is made more precious by the instabilities in sampling. It is hard to calculate when the actual value of an average comes out in fractions. It is not appropriate for sociological study.

\subsection{The Derivation of the Study:}

The derivation of this study can be explained as follows. In the beginning, we choose the maximum values of each maximized objective function and the minimum values of each minimized objective function by substituting corner points without zero point in the convex feasible region (6) or (8) in the fractional objectives.

$\therefore \operatorname{Max} . z_{i}=x_{n}^{*}=\theta_{i}$, at $u_{i}=\left(x_{1}^{*}, x_{2}^{*}, \ldots, x_{n-1}^{*}\right) i=1, \ldots, r$, are maximum values

and Min. $z_{k}=x_{n}^{*}=\emptyset_{k}$, at $v_{k}=\left(x_{1}^{*}, x_{2}^{*}, \ldots, x_{n-1}^{*}\right) k=r+1, \ldots, s$, are minimum values.

Where, $u_{i}$ and $v_{k} \in S i=1, \ldots, r$ and $k=r+1, \ldots, s$, are corner points, and $S=\left\{x \in R^{n}: A x \leq b, x \geq 0\right\}$, then by definition (3.4)(Max or Min) objective functions $z_{i}, i=1, \ldots, s$. has continuous at $u_{i}$ and $v_{k}, i=1, \ldots, r, k=$ $r+1, \ldots, s$, and it is differentiable. After that, by using formula (3), we get:

$z_{i}-\theta_{i}=M_{x 1}\left(x_{1}-x_{1}^{*}\right)+M_{x 2}\left(x_{2}-x_{2}^{*}\right)+\cdots+M_{x(n-1)}\left(x_{n-1}-x_{(n-1) 0}\right)$, at $u_{i}=\left(x_{1}^{*}, x_{2}^{*}, \ldots, x_{n-1}^{*}\right), i=1, \ldots, r$

$z_{k}-\emptyset_{k}=M_{x 1}\left(x_{1}-x_{1}^{*}\right)+M_{x 2}\left(x_{2}-x_{2}^{*}\right)+\cdots+M_{x(n-1)}\left(x_{n-1}-x_{(n-1) 0}\right)$, at $v_{k}=\left(x_{1}^{*}, x_{2}^{*}, \ldots, x_{n-1}^{*}\right), k=r+1, \ldots, s$

And we obtain the multi-linear function.

$$
\begin{aligned}
& z_{i}=a_{1} x_{1}+a_{2} x_{2}+\cdots+a_{n-1} x_{n-1}+e, i=1, \ldots, r, \text { and } z_{k}=b_{1} x_{1}+b_{2} x_{2}+\cdots+b_{n-1} x_{n-1}+t, k=r+1, \ldots, s \\
& \text { Where, } a_{1}=M_{x 1}=\frac{\partial z_{i}}{\partial x_{1}}, \ldots, a_{n-1}=M_{x(n-1)}=\frac{\partial z_{i}}{\partial x_{n-1}} \\
& e=\theta_{i}-\left(M_{x 1} x_{1}^{*}+\cdots+M_{x(n-1)} x_{n-1}^{*}\right) \text { according to } u_{i}=\left(x_{1}^{*}, x_{2}^{*}, \ldots, x_{n-1}^{*}\right), \quad i=1, \ldots, r, \\
& \text { And, } b_{1}=M_{x 1}=\frac{\partial z_{i}}{\partial x_{1}}, \ldots, b_{n-1}=M_{x(n-1)}=\frac{\partial z_{i}}{\partial x_{n-1}}, \\
& t=\emptyset_{k}-\left(M_{x 1} x_{1}^{*}+\cdots+M_{x(n-1)} x_{n-1}^{*}\right) \text { according to } v_{k}=\left(x_{1}^{*}, x_{2}^{*}, \ldots, x_{n-1}^{*}\right), k=r+1, \ldots, s .
\end{aligned}
$$

The techniques convert MOLPP into SOLPP, for each linear objective in (10) will be solved by the simplex method individually. Then we obtain the values, $z_{i}=\gamma_{i}, i=1, \ldots, r$, and $z_{k}=\varepsilon_{k}, k=r+1, \ldots, s$ after that, calculate the mean deviation using formula (4) of $\gamma_{i}, i=1, \ldots, r$, we get, $M D_{1}=\frac{\sum_{i=1}^{r}\left(\gamma_{i}-\bar{\gamma}\right)}{r}$, where $\bar{\gamma}=\frac{\sum_{i=1}^{r} \gamma_{i}}{r}$ 
Also, same calculate of $\varepsilon_{k}, k=r+1, \ldots, s$, we get, $M D_{2}=\frac{\sum_{k=r+1}^{s}\left(\varepsilon_{k}-\bar{\varepsilon}\right)}{s-r}$, where $\bar{\varepsilon}=\frac{\sum_{k=r+1}^{s} \varepsilon_{k}}{s-r}$

Let, $z_{1}^{*}=\sum_{i=1}^{r} z_{i}$, and $z_{2}^{*}=\sum_{i=r+1}^{s} z_{k}$

Then, we suggest two techniques for process of the converting MOLPP into LPP.

\section{1- New Mean Deviation(NMD) Technique:}

$\operatorname{Max} . z^{*}=\frac{z_{1}^{*}-z_{2}^{*}}{N M D}$

where, $N M D=\frac{M D_{1}+M D_{2}}{R}, R=\frac{\text { number of objectives }}{\text { types of objectives }}$

2- Advanced Mean Deviation(AMD) Technique:

$\operatorname{Max} . z^{*}=\frac{z_{1}^{*}-z_{2}^{*}}{A M D}$.

Where, $A M D=\frac{M D_{1}+M D_{2}}{s}, \mathrm{~s}$ is number of objectives.

There are more details about the values of $\gamma_{i}, i=1, \ldots, r$, and $\varepsilon_{k}, k=r+1, \ldots, s$, so we have four cases.

In the first case, if one or more of each values $\gamma_{i}, i=1, \ldots, r$, and $\varepsilon_{k}, k=r+1, \ldots, s$, are different from each other's, then $M D_{1} \& M D_{2}$ will not equal to zero. It is illustrated in equations (13), (14). In the second case, if $\gamma_{1}=\gamma_{2}=\cdots=$ $\gamma_{r} \neq 0$ or $\varepsilon_{r+1}=\varepsilon_{r+2}=\cdots=\varepsilon_{s} \neq 0$, then, $M D_{1}$, or $M D_{2}$, will be zero, that is not an issue. We can use the same previous equations (13), (14). But in the third case, if $\gamma_{1}=\gamma_{2}=\cdots=\gamma_{r} \neq 0$ is equal and $\varepsilon_{r+1}=\varepsilon_{r+2}=\cdots=\varepsilon_{s} \neq$ 0 is equal, then $M D_{1}, \& M D_{2}$, it will be zero, we have to mention; $M a x \cdot z^{*}=\frac{z_{1}^{*}}{\gamma}-\frac{z_{2}^{*}}{|\varepsilon|}$. In the last case, if $\gamma_{1}=\gamma_{2}=$ $\cdots=\gamma_{r}=\varepsilon_{r+1}=\varepsilon_{r+2}=\cdots=\varepsilon_{s} \neq 0$, then $M D_{1} \& M D_{2}$ will be zero, therefore $\operatorname{Max} . z^{*}=\frac{z_{1}^{*}-z_{2}^{*}}{\gamma}$.

Note that, if the number of minimized objective functions of the equation (5) or (7) is equal to zero then, which is the same situation as in the second case $M D_{2}=0$.

$\operatorname{Max} \cdot z^{*}=\frac{z_{1}^{*}}{N M D^{\prime}}$, where $N M D=\frac{M D_{1}}{R}$, and $M a x \cdot z^{*}=\frac{z_{1}^{*}}{A M D^{\prime}}$, where $A M D=\frac{M D_{1}}{s}$

Otherwise, the same situation will happen if the number of maximized objective functions of the equation (5) or (7) is equal to zero $M D_{1}=0$. Then, $M a x \cdot z^{*}=\frac{-z_{2}^{*}}{N M D^{2}}$, where $N M D=\frac{M D_{2}}{R}$ and $M a x \cdot z^{*}=\frac{-z_{2}^{*}}{A M D^{\prime}}$, where $A M D=\frac{M D_{2}}{s}$

Finally, Max. $z^{*}$ subject to the same constraint (6) or (8) will be SOLPP, solving this problem by the simplex routine. Because this SOLPP is exact equivalent weighted and has an efficient solution to basic MOFPP, it is thought to have the same properties as the objective functions.

\subsection{Algorithm:}

This algorithm is to get the optimal solution for the MOFPP that we cleared previously; it can be briefed as follows.

Step1: Determine nonzero corner points in the feasible region.

Step2: Find maximum values of each maximized objective functions; named $\theta_{i}, i=1, \ldots, r$ and minimum values of each minimized objective functions, named $\emptyset_{k}, k=r+1, \ldots, s$. by substituting corner points in step1 on objective function (5) and (7).

Step3: Select points $u_{i}=\left(x_{1}^{*}, x_{2}^{*}, \ldots, x_{n-1}^{*}\right), i=1, \ldots, r$, of maximum values and $v_{k}=\left(x_{1}^{*}, x_{2}^{*}, \ldots, x_{n-1}^{*}\right), k=r+$ $1, \ldots, s$, of minimum values

Step4: Transform each fractional objective function into linear objective functions by formula (9) at the points in step3; we obtain equations (10).

Step5: Solve each objective function in (10) subject to the constraint (6) or (8) by simplex method. 
Step6: Test feasibility of optimal solution obtains in step5, if it's infeasible, use dual simplex method to avoid infeasibility, otherwise go to step7.

Step7: Assign each optimal values of objective functions obtain in step6, $z_{i}=\gamma_{i}, i=1, \ldots, r$ and $z_{k}=\varepsilon_{k}, k=r+1, \ldots, s$.

Step8: Calculate $M D_{1} \& M D_{2}$, using formula (11) and (12).

Step9: Construct the single objective function which has formula (13), (14).

Step10: Optimize the single objective function subject to the same basic constraint (6) or (8).

\subsection{Flow Chart:}

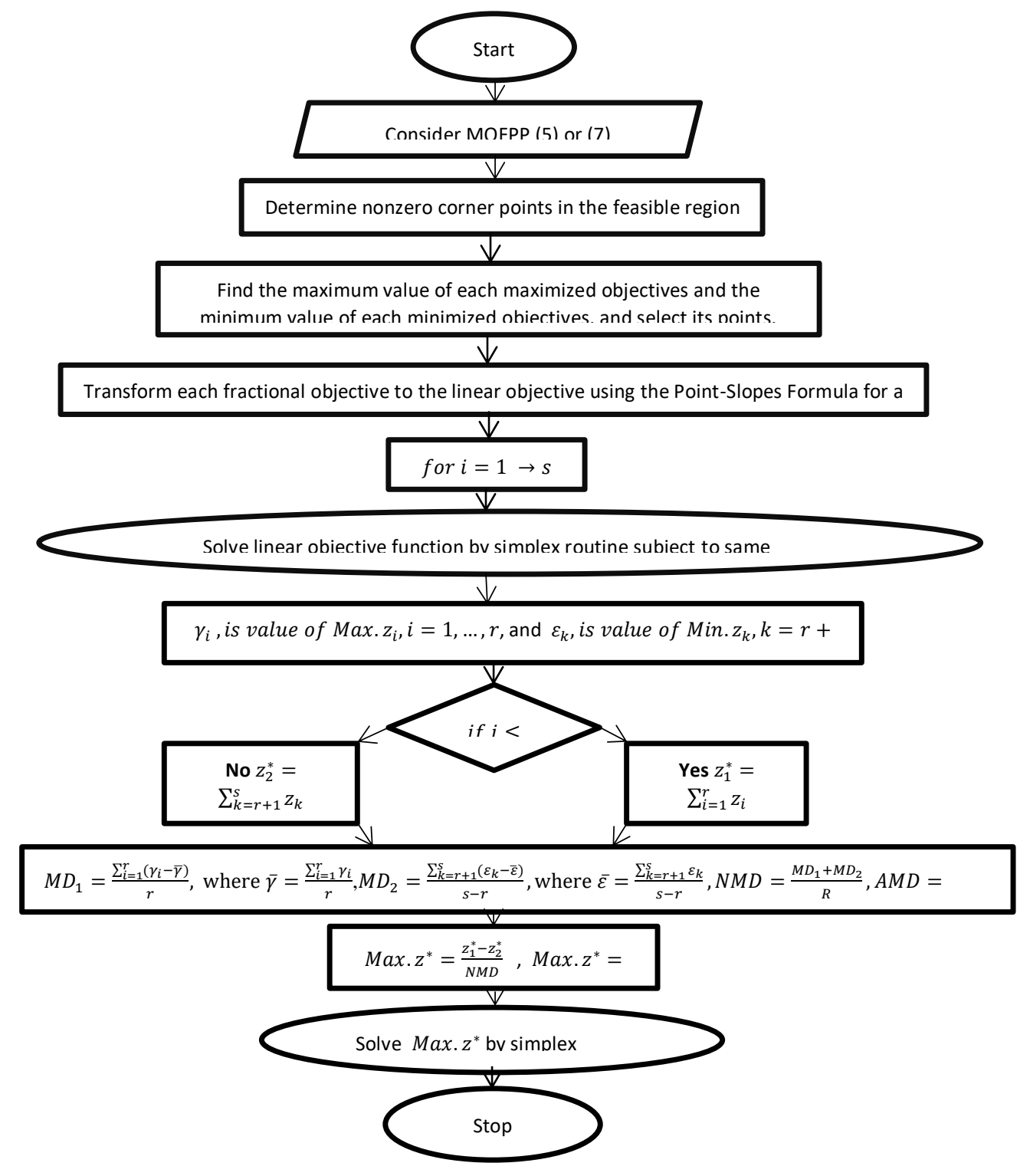

Figure1: Flow Chart 


\section{Numerical Examples:}

\section{Example 1:}

$\operatorname{Max} . Z_{1}=\frac{\left(2 x_{1}+x_{2}+1\right)\left(2 x_{1}+x_{2}+2\right)}{\left(2 x_{1}+2 x_{2}+2\right)}, \operatorname{Max} . Z_{2}=\frac{\left(4 x_{1}+2 x_{2}+2\right)\left(6 x_{1}+3 x_{2}+6\right)}{\left(3 x_{1}+3 x_{2}+3\right)}, \operatorname{Max} . Z_{3}=\frac{\left(4 x_{1}+2 x_{2}+2\right)\left(6 x_{1}+3 x_{2}+6\right)}{\left(6 x_{1}+6 x_{2}+6\right)}$

Min. $Z_{4}=\frac{\left(-8 x_{1}-4 x_{2}-4\right)\left(6 x_{1}+3 x_{2}+6\right)}{\left(5 x_{1}+5 x_{2}+5\right)}$, Min. $Z_{5}=\frac{\left(-4 x_{1}-2 x_{2}-2\right)\left(10 x_{1}+5 x_{2}+10\right)}{\left(2 x_{1}+2 x_{2}+2\right)}$

Subject to:

$x_{1}+2 x_{2} \leq 4$

$3 x_{1}+x_{2} \leq 6$

$x_{1}, x_{2} \geq 0$

Solution:

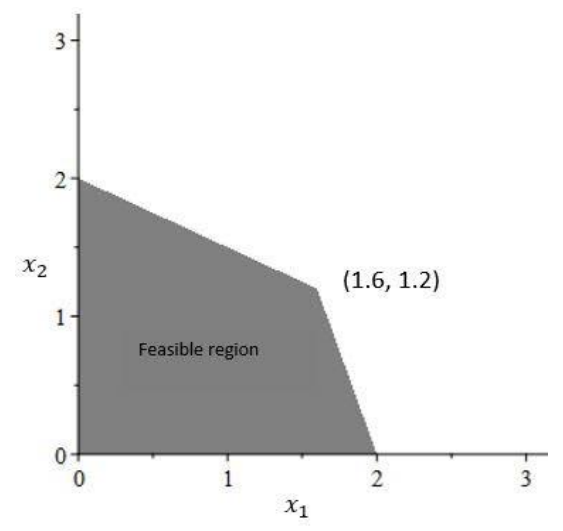

Figure2: Constraints (15)

The corner points without $(0,0)$ on the feasible region in figure 2 are $\{(2,0),(0,2)$ and $(1.6,1.2)\}$

It is observed that, $\theta_{1}=5, \theta_{2}=20, \theta_{3}=10$ are maximum values and $\emptyset_{4}=-24, \emptyset_{5}=-50$ are minimum values. And $u_{1}=u_{2}=u_{3}=v_{1}=v_{2}=(2,0)$,

Through using formula (9) for each quadratic fractional objective function, we obtain the multi linear form,

$z_{1}=2 x_{1}+0.1666 x_{2}+1$

$z_{2}=8 x_{1}+0.6666 x_{2}+4$

$z_{3}=4 x_{1}+0.3333 x_{2}+2$

$z_{4}=-9.6 x_{1}-0.8 x_{2}-4.8$

$z_{5}=-20 x_{1}-1.6666 x_{2}-10$

Now, $z_{i}, \mathrm{i}=1, \ldots, 5$, subject to constraint (15), is called MOLPP. Solving each $z_{i}, \mathrm{i}=1, \ldots, 5$, subject to (15) by simplex method, we obtain the values $\gamma_{1}=5, \gamma_{2}=20, \gamma_{3}=10, \varepsilon_{4}=-24, \varepsilon_{5}=-50$.

After that, $z_{1}^{*}=z_{1}+z_{2}+z_{3}, z_{2}^{*}=z_{4}+z_{5}$ and $z^{*}=z_{1}^{*}-z_{2}^{*}$

$\therefore z^{*}=43.6 x_{1}+3.6325 x_{2}+21.8$ 
Then, use formulas (11) \& (12) to find mean deviations.

$M D_{1}=5.5555, M D_{2}=13, N M D=7.4222$, and $A M D=3.7111$,

Here, we apply the two techniques that were created by us:

New Mean Deviation Technique:

$\operatorname{Max} . z=\frac{z^{*}}{N M D}$

$\therefore \operatorname{Max} . z=5.8742 x_{1}+0.4994 x_{2}+2.9371$

Subject to constraint (15), we obtained the optimal solution.

$x_{1}=2, x_{2}=0$ and Max. $z=14.6855$

\section{Advanced Mean Deviation Technique:}

$\operatorname{Max} . z=\frac{z^{*}}{A M D}$

$\therefore$ Max. $z=11.7485 x_{1}+0.9788 x_{2}+5.8742$

Subject to constraint (15), we obtained the optimal solution.

$x_{1}=2, x_{2}=0$ and Max. $z=29.3712$

Next, we calculate some other techniques used in this study to solve equation (16) as below:

\section{Chandra Sen Technique:}

$\operatorname{Max} . Z=\sum_{i=1}^{r} \frac{z_{i}}{\left|\gamma_{i}\right|}-\sum_{k=r+1}^{s} \frac{z_{k}}{\left|\varepsilon_{k}\right|}$

$\therefore \operatorname{Max} . z=2 x_{1}+0.1666 x_{2}+1$

Subject to constraint (15), we obtained the optimal solution.

$x_{1}=2, x_{2}=0$ and Max. $z=5$

\section{Advanced Optimal Average Technique:}

$\operatorname{Max} . Z=\frac{\sum_{i=1}^{r} z_{i}-\sum_{k=r+1}^{S} z_{k}}{A O_{A v}}$, Where, $A O_{A v}=\frac{m_{1}+m_{2}}{s}$

$\therefore \operatorname{Max} . z=7.5172 x_{1}+0.6262 x_{2}+3.7586$

Subject to constraint (15), we obtained the optimal solution.

$x_{1}=2, x_{2}=0$ and $\operatorname{Max} . z=18.793$

Advanced Harmonic Average Technique:

$\operatorname{Max} . Z=\frac{\sum_{i=1}^{r} z_{i}-\sum_{k=r+1}^{S} z_{k}}{A H_{a v}}$, Where $A H_{a v}=\frac{2\left|m_{1}\right|\left|m_{2}\right|}{\left|m_{1}\right|+\left|m_{2}\right|}$

$m_{1}=\min \left(\left|\gamma_{i}\right|\right), i=1, \ldots, r, m_{2}=\min \left(\left|\varepsilon_{k}\right|\right), k=r+1, \ldots, s$ 
$\therefore \operatorname{Max} . z=5.2683 x_{1}+0.4389 x_{2}+2.6341$

Subject to constraint (15), we obtained the optimal solution.

$x_{1}=2, x_{2}=0$ and Max. $z=13.1707$

\section{Example 2:}

$\operatorname{Max} . Z_{1}=\frac{3 x_{1}-2 x_{2}}{x_{1}+x_{2}+1}, \operatorname{Max} . Z_{2}=\frac{9 x_{1}+3 x_{2}}{x_{1}+x_{2}+1}, \operatorname{Max} . Z_{3}=\frac{3 x_{1}+5 x_{2}}{2 x_{1}+2 x_{2}+2}$

Min. $Z_{4}=\frac{-6 x_{1}+2 x_{2}}{2 x_{1}+2 x_{2}+2}, \operatorname{Min} \cdot Z_{5}=\frac{-3 x_{1}-x_{2}}{x_{1}+x_{2}+1}$

Subject to:

$x_{1}+x_{2} \leq 2$

$9 x_{1}+x_{2} \leq 9$

$x_{1}, x_{2} \geq 0$

Solution:

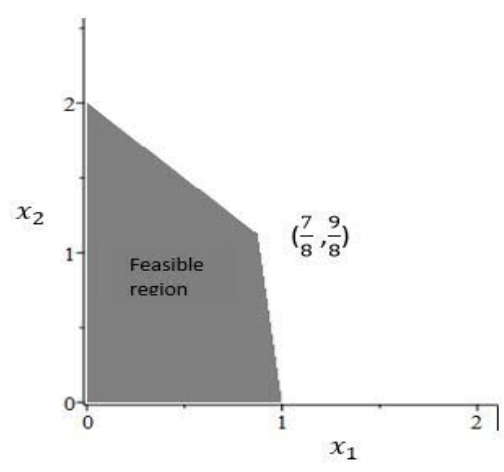

Figuer3: Constraints (17)

The corner points without $(0,0)$ on the feasible region in figure 3 are $\{(1,0),(0,2)$ and $(7 / 8,9 / 8)\}$

It is cleared that, $\theta_{1}=1.5, \theta_{2}=4.5, \theta_{3}=0.75, \emptyset_{4}=-1.5, \emptyset_{5}=-1.5$, and $u_{1}=u_{2}=u_{3}=v_{1}=v_{2}=(1,0)$.

Through using formula (9), for each linear fractional objective function, we obtain the multi linear form,

$z_{1}=0.75 x_{1}-1.75 x_{2}+0.75$

$z_{2}=2.25 x_{1}-0.75 x_{2}+2.25$

$z_{3}=0.375 x_{1}-1.625 x_{2}+0.375$

$z_{4}=-0.75 x_{1}+1.25 x_{2}-0.75$

$z_{5}=-0.75 x_{1}+0.25 x_{2}-0.75$

Now, solving each $z_{i}, \mathrm{i}=1, \ldots, 5$, subject to (17) by simplex method, we obtain the values $\gamma_{1}=1.5, \gamma_{2}=4.5, \gamma_{3}=$ $0.75, \varepsilon_{4}=-1.5, \varepsilon_{5}=-1.5$.

After that, $z_{1}^{*}=z_{1}+z_{2}+z_{3}, z_{2}^{*}=z_{4}+z_{5}$ and $z^{*}=z_{1}^{*}-z_{2}^{*}$ 
$\therefore z^{*}=4.875 x_{1}-5.625 x_{2}+4.875$

Then, use formulas (11) \& (12) to find mean deviations. $M D_{1}=1.5, M D_{2}=0, N M D=0.6$, and $A M D=0.3$

Here, we apply the two techniques that were created by us:

New Mean Deviation Technique:

$\therefore \operatorname{Max} . z=8.125 x_{1}-9.375 x_{2}+8.125$

Subject to constraint (17), we obtained the optimal solution.

$x_{1}=1, x_{2}=0$ and Max. $z=16.25$

\section{Advanced Mean Deviation Technique:}

$\therefore \operatorname{Max} . z=16.25 x_{1}-18.75 x_{2}+16.25$

Subject to constraint (17), we obtained the optimal solution.

$x_{1}=1, x_{2}=0$ and Max. $z=32.5$

Next, we calculate some other techniques used in this study, to solve equation (18) as below:

\section{Chandra Sen Technique:}

$\therefore \operatorname{Max} . z=2.5 x_{1}-4.497 x_{2}+2.5$

Subject to constraint (17), we obtained the optimal solution.

$x_{1}=1, x_{2}=0$ and Max. $z=5$

\section{Advanced Optimal Average Technique:}

$\therefore \operatorname{Max} . z=10.8333 x_{1}-12.5 x_{2}+10.8333$

Subject to constraint (17), we obtained the optimal solution.

$x_{1}=1, x_{2}=0$ and Max. $z=21.6666$

\section{Advanced Harmonic Average Technique:}

$\therefore \operatorname{Max} . z=4.875 x_{1}-5.625 x_{2}+4.875$

Subject to constraint (17), we obtained the optimal solution.

$x_{1}=1, x_{2}=0$ and Max. $z=9.75$

Example 3:(Lachhwani, 2017)

$\operatorname{Max} . Z_{1}=\frac{\left(2 x_{1}+20 x_{2}+12\right)\left(x_{1}+10 x_{2}+17\right)}{\left(-2 x_{1}-5 x_{2}+15\right)\left(2 x_{1}+5 x_{2}+11\right)}, \operatorname{Max} . Z_{2}=\frac{\left(2 x_{1}+20 x_{2}+12\right)\left(3 x_{1}+30 x_{2}+51\right)}{\left(-4 x_{1}-10 x_{2}+30\right)\left(2 x_{1}+5 x_{2}+11\right)}$

Subject to:

$x_{1}+15 x_{2} \leq 2$

$3 x_{1}+20 x_{2} \leq 4$

$x_{1}, x_{2} \geq 0$ 
Solution:

The corner points without $(0,0)$ on the feasible region are $\{(0,0.13),(1.3,0)$ and $(0.8,0.08)\}$

It is clear that, $\theta_{1}=1.6728, \theta_{2}=2.5093$, and $u_{1}=u_{2}=(0.8,0.08)$

Through using formula (9), for each quadratic fractional objective function, we obtain the multi linear form,

$z_{1}=0.31 x_{1}+3.1 x_{2}+1.1768$

$z_{2}=0.465 x_{1}+4.65 x_{2}+1.7653$

Now, solving each $z_{i}, \mathrm{i}=1,2$, subject to (19) by simplex method, we obtain the values $\gamma_{1}=1.6728, \gamma_{2}=2.5093$.

After that, $z_{1}^{*}=z_{1}+z_{2}, z_{2}^{*}=0$ and $z^{*}=z_{1}^{*}-z_{2}^{*}$

$\therefore z^{*}=0.775 x_{1}+7.75 x_{2}+2.9421$

Then, use formulas (11) \& (12) to find mean deviations.

$M D_{1}=0.4182, M D_{2}=0, N M D=0.2091$, and $A M D=0.2091$

Here, we apply the techniques that were created by us:

Since $N M D=A M D=0.2091$, then,

$\therefore \operatorname{Max} . z=3.7 x_{1}+37.06 x_{2}+14.07$

Subject to constraint (19), we obtained the optimal solution.

$x_{1}=0.08, x_{2}=0.08$ and $\operatorname{Max} . z=19.9948$

This example has the same optimal solution as that of Lachhwani.

$x_{1}=0.08, x_{2}=0.08$.

Example 4:(Borza \& Rambely, 2021; Chakraborty \& Gupta, 2002)

$\operatorname{Max} . Z_{1}=\frac{-3 x_{1}+2 x_{2}}{x_{1}+x_{2}+3}, \operatorname{Max} . Z_{2}=\frac{7 x_{1}+2 x_{2}}{5 x_{1}+2 x_{2}+1}$

Subject to:

$x_{1}-x_{2} \geq 1$

$2 x_{1}+3 x_{2} \leq 15$

$x_{1} \geq 3$

$x_{1}, x_{2} \geq 0$

Solution:

The corner points on the feasible region are $\{(3,2),(3,0),(3.6,2.6)$ and $(7.5,0)\}$

It is cleared that, $\theta_{1}=-0.6086, \theta_{2}=1.3636$, and $u_{1}=(3.6,2.6), u_{2}=(7.5,0)$.

Through using formula (9), for each linear fractional objective function, we obtain the multi linear form,

$z_{1}=-0.2599 x_{1}+0.2835 x_{2}-0.41$ 
$z_{2}=0.004722 x_{1}-0.04486 x_{2}+1.2382$

Now, solving each $z_{i}, \mathrm{i}=1,2$ subject to (21) by simplex method, we obtain the values $\gamma_{1}=-0.60854, \gamma_{2}=$ 1.273615 .

After that, $z_{1}^{*}=z_{1}+z_{2}, z_{2}^{*}=0$ and $z^{*}=z_{1}^{*}-z_{2}^{*}$

$\therefore z^{*}=-0.255178 x_{1}+0.23864 x_{2}+0.9182$

Then, use formulas (11) \& (12) to find mean deviations. $M D_{1}=0.941, M D_{2}=0, N M D=0.3136$, and $A M D=$ 0.3136

Here, we apply our techniques that were created by us:

Since $N M D=A M D=0.3136$, then,

$\therefore \operatorname{Max} . z=-0.8137 x_{1}+0.7609+2.9279$

Subject to constraint (21), we obtained the optimal solution.

$x_{1}=3, x_{2}=2$ and Max. $z=2.0086$.

This example has the same optimal solution as that of Chakraborty and Gupta.

$x_{1}=3, x_{2}=2, \operatorname{Max} \cdot z_{1}=-0.625$, and $\operatorname{Max} \cdot z_{2}=1.15$

\section{Discussion and Comparison:}

It is clear from the results of the examples above that our technique is efficient in solving MOFPP. The solutions of MOFPP in examples 1, 2 indicate that Chnadra sen, Advanced optimal average, and Advanced harmonic average are incapable of providing the cooperating solution. In Table 1, it is clear that the results obtained when using NMD and AMD are better than the other results. In examples 3, 4 Chakraborty \& Gupta, and Lachhwani are difficult to solve and do not dominate our proposed solution. The ordinary of association functions shows that our suggested technique has a better efficiency. The comparison of the set of techniques is based on the values of the objective functions. After solving the numerical examples, we found the Max.Z. One of the features of our proposal, is to convert any fractional programming into linear programming under some limitations, such as this method cannot apply to the coefficients of variable objective functions that are not fixed numbers (rough interval, grey interval, or fuzzy number), but this issue is to be treated by using some of the definitions and theorems in advanced differential equations in the future.

Comparison of the numerical results which are attained from previous examples 1 and 2 is presented in the following Table 1:

Table 1

\begin{tabular}{llll}
\hline No. & Techniques & Example1 & Example2 \\
\hline 1 & Chandra Sen & 5 & 5 \\
2 & Advanced Optimal Average & 18.793 & 21.6666 \\
3 & Advanced Harmonic Average & 13.1707 & 9.75 \\
4 & New Mean Deviation & 14.6855 & 16.25 \\
5 & Advanced Mean Deviation & 29.3712 & 32.5 \\
\hline
\end{tabular}

\section{Conclusion:}

In this paper, we presented a new technique to solve the MOFPP. In the approach, the MOFPP was finally transformed into a LPP using some suitable formula for each fractional objective function in the feasible region. MOFPP is reduced to MOLPP, and MOLPP is reduced to SOLPP.The obtained LPP is solved by considering (NMD\& AMD) of the weights in the linear objective functions. The proposed solution to MOFPP continually produces efficient solutions and to the difficulty of solving MOFPP, our method has a summary easy 
calculation. Four examples are explained to show the approach. Our technique is a very fast way to get solutions to particular situations in real-life problems, and it gives better outcomes compared to Chandra sen, advanced optimal average, advanced harmonic average, and smooth calculation according to Chakraborty and Gupta, Pramy and Islam, Borza and Rambely, and Lachhwani.

\section{References}

Akhtar, H., \& Modi, G. (2017). An approach for solving multi-objective linear fractional programming problem and it's comparison with other techniques. Intearnational Journal of Scientific and Innovative Mathematical Reasearch (IJSIMR), 5(11).

Akhtar, H., Modi, G., \& Duraphe, S. (2017). Transforming and optimizing multi-objective quadratic fractional programming problem. International Journal of Statistics and Applied Mathematics, 2(1), 01-05.

Borza, M., \& Rambely, A. S. (2021). A New Method to Solve Multi-Objective Linear Fractional Problems. Fuzzy Information and Engineering, 1-12.

Chakraborty, M., \& Gupta, S. (2002). Fuzzy mathematical programming for multi objective linear fractional programming problem. Fuzzy sets and systems, 125(3), 335-342.

Dinkelbach, W. (1967). On nonlinear fractional programming. Management science, 13(7), 492-498.

Gupta, D., Rani, N., \& Goyal, V. (2019). On solving Multi-objective Quadratic Fractional Optimization Model with Asymmetric Trapezoidal Fuzzy Number.

Güzel, N. (2013). A proposal to the solution of multiobjective linear fractional programming problem. Abstract and applied analysis,

Hejazi, M. A., \& Nobakhtian, S. (2020). Optimality conditions for multiobjective fractional programming, via convexificators. Journal of Industrial \& Management Optimization, 16(2), 623.

Lachhwani, K. (2017). On FGP approach to multiobjective quadratic fractional programming problem. International Journal of Applied and Computational Mathematics, 3(4), 3443-3453.

Mehdi, M. A., Chergui, M. E.-A., \& Abbas, M. (2014). An improved method for solving multiobjective integer linear fractional programming problem. Advances in Decision Sciences, 2014.

Nayak, S., \& Ojha, A. K. (2019). Solution approach to multi-objective linear fractional programming problem using parametric functions. Opsearch, 56(1), 174-190.

Pramy, F. A., \& Islam, M. A. (2017). Determining efficient solutions of multi-objective linear fractional programming problems and application. Open Journal of Optimization, 6(04), 164.

Sen, C. (1983). A new approach for multi-objective rural development planning. The Indian Economic Journal, 30(4), 91-96.

Sen, C. (2020). Improved averaging techniques for solving multi-objective optimization (MOO) problems. SN Applied Sciences, 2(2), 1-6.

Sivri, M., Albayrak, I., \& Temelcan, G. (2018). A novel approach for solving quadratic fractional programming problems. Croatian Operational Research Review, 9(2), 199.

Sulaiman, N. A., \& Mustafa, R. B. (2016). Using harmonic mean to solve multi-objective linear programming problems. American journal of operations Research, 6(1), 25-30.

Suleiman, N. A., \& Nawkhass, M. A. (2013). Transforming and solving multi-objective quadratic fractional programming problems by optimal average of maximin \& minimax techniques. American Journal of Operational Research, 3(3), 92-98.

Valipour, E., Yaghoobi, M., \& Mashinchi, M. (2014). An iterative approach to solve multiobjective linear fractional programming problems. Applied Mathematical Modelling, 38(1), 38-49. 